\title{
Refined counting of core partitions into $d$-distinct parts
}

\author{
Hannah E. Burson \\ School of Mathematics \\ University of Minnesota \\ Minneapolis, MN, U.S.A. \\ hburson@umn. edu
}

\author{
Simone Sisneros-Thiry \\ Department of Mathematics \\ California State University, East Bay \\ Hayward, CA, U.S.A. \\ simone.sisnerosthiry@csueastbay.edu
}

\author{
Armin Straub \\ Department of Mathematics and Statistics \\ University of South Alabama \\ Mobile, AL, U.S.A. \\ straub@southalabama.edu
}

Submitted: Jun 22, 2020; Accepted: Jan 13, 2021; Published: Feb 12, 2021

(C) The authors. Released under the CC BY license (International 4.0).

\begin{abstract}
Using a combinatorial bijection with certain abaci diagrams, Nath and Sellers have enumerated $(s, m s \pm 1)$-core partitions into distinct parts. We generalize their result in several directions by including the number of parts of these partitions, by considering $d$-distinct partitions, and by allowing more general $(s, m s \pm r)$-core partitions. As an application of our approach, we obtain the average and maximum number of parts of these core partitions.
\end{abstract}

Mathematics Subject Classifications: 05A17, 11P57

\section{Introduction}

If $\lambda_{1} \geqslant \lambda_{2} \geqslant \cdots \geqslant \lambda_{r} \geqslant 1$, where the parts $\lambda_{i}$ are integers, then $\lambda=\left(\lambda_{1}, \lambda_{2}, \ldots, \lambda_{r}\right)$ is an (integer) partition of $|\lambda|=\lambda_{1}+\cdots+\lambda_{r}$. Its number of parts is $n(\lambda)=r$. Excellent introductions to partitions include [3] and [4]. A partition is $d$-distinct if its parts differ by at least $d$. In the case $d=1$, these are precisely partitions into distinct parts. The Young diagram of a partition $\lambda$ is a left-justified array of square cells, where the first row contains $\lambda_{1}$ cells, the second row $\lambda_{2}$ cells, and so on. The hook assigned to each cell $u$ consists of the cell $u$ itself as well as all cells below $u$ and to the right of $u$. The length of 
a hook is the number of cells it contains. A partition $\lambda$ is said to be $t$-core if there is no hook in its Young diagram that has length $t$. For an introduction to this notion we refer to, for instance, [5]. More generally, $\lambda$ is said to be $\left(t_{1}, t_{2}, \ldots, t_{m}\right)$-core if $\lambda$ is $t_{i}$-core for all $i \in\{1,2, \ldots, m\}$.

In this paper, we are concerned with counting certain core partitions. This line of inquiry has received increasing interest since Anderson [2] proved that the number of $(s, t)$-core partitions is

$$
\frac{1}{s+t}\left(\begin{array}{c}
s+t \\
s
\end{array}\right)
$$

if $s$ and $t$ are coprime (otherwise, there are infinitely many such partitions). It remains an open problem to similarly enumerate $(s, t)$-core partitions into distinct parts. Towards that problem, it was shown in [18] that $(s, m s-1)$-core partitions into distinct parts are counted by Fibonacci-like numbers. This count was generalized by Nath and Sellers [13] to also include $(s, m s+1)$-core partitions. Following [13], $(s, m s \pm 1)$-core partitions refer to $(s, m s-1)$-core or $(s, m s+1)$-core partitions.

Theorem 1. Let $m, s \geqslant 1$. The number $C_{s, m}^{ \pm}$of $(s, m s \pm 1)$-core partitions into distinct parts is characterized by

$$
C_{s, m}^{ \pm}=C_{s-1, m}^{ \pm}+m C_{s-2, m}^{ \pm}
$$

for $s \geqslant 3$, with the initial conditions $C_{1, m}^{ \pm}=1, C_{2, m}^{-}=m$ and $C_{2, m}^{+}=m+1$.

In the case $m=1$, the numbers $C_{s, 1}^{-}=F_{s}$ are the familiar Fibonacci numbers. This special case was predicted by Amdeberhan [1] and also independently proved by Xiong [19].

Nath and Sellers [13] proved (the case of $(s, m s+1)$-core partitions of) Theorem 1 combinatorially by viewing the partitions as certain abaci. This correspondence will be introduced in Section 2. Building on their combinatorial approach, our main result extends Theorem 1 in various directions:

(a) we keep track of the number of parts of the involved partitions,

(b) we consider partitions into $d$-distinct parts (which, in the case $d=1$, are partitions into distinct parts),

(c) we allow $(s, m s+r)$-core partitions for certain integers $r$, and

(d) we consider $s$-core partitions with maximum hook length $m s+r$ for all $m, r \in \mathbb{Z}$.

To that end, let $\mathcal{C}_{s, m, r}^{d}$ be the set of $(s, m s+r)$-core partitions into $d$-distinct parts. We denote with

$$
\boldsymbol{C}_{s, m, r}^{d}(q)=\sum_{\lambda \in \mathcal{C}_{s, m, r}^{d}} q^{n(\lambda)}
$$

the generating polynomial for the number of parts of the partitions in $\mathcal{C}_{s, m, r}^{d}$. If $d$ is omitted in the notation, it is implicit that $d=1$. Observe that $C_{s, m}^{ \pm}=\boldsymbol{C}_{s, m, \pm 1}(1)$. 
Theorem 2. Let $d, m, r \geqslant 1$. Write $f_{s}(q)=C_{s, m, r}^{d}(q)$. If $s>d+1$ and $r \leqslant d$, then

$$
f_{s}(q)=f_{s-1}(q)+\left(q+q^{2}+\ldots+q^{m}\right) f_{s-d-1}(q) .
$$

If $r=-1$, then the same conclusion holds provided that $s>2$, if $d=1$, and $s>2 d+1$, if $d>1$.

We provide initial conditions, which specialize to the ones in Theorem 1, in Lemma 18. In light of Lemma 6, we prove Theorem 2 as a special case of our Theorems 12 and 14 (as well as Lemma 15, in the case $r=-1$ and $s=2 d+2$, and Lemma 17 in the case $r=-1$ and $d=1$ ) on certain abaci. In fact, it follows from Theorem 12 that the condition $r \leqslant d$ can be dropped if $\boldsymbol{C}_{s, m, r}^{d}(q)$ is modified to be the generating polynomial of parts of $s$-core partitions into $d$-distinct parts with largest hook of length less than $m s+r$. Additionally, in Theorem 14, we provide an extension to negative values $r<-1$.

Theorem 2 unifies several results in the recent literature: as indicated, the special cases $(d, r, q)=(1, \pm 1,1)$ result in Theorem 1 by Straub [18] and Nath and Sellers [13]. More generally, the case $(d, r)=(1,1)$ has been established by Paramonov [15], who notes that the approach applies to the case $(d, r)=(1,-1)$ as well. In the cases $(m, q)=(1,1)$, we obtain results on $d$-distinct partitions by Sahin [16], which were generalized by Kravitz [12] who also considers the case $m>1$.

In Theorems 12 and 14, we show that the enumeration in Theorem 2 extends naturally to $s$-core abaci of bounded height. In Section 2, we review the combinatorial correspondence between core partitions and abaci, and prove several preliminary results. Let us note, for instance, that Nath and Sellers [13] prove the case $(d, r, q)=(1,1,1)$ in a combinatorial manner and then use an algebraic argument based on generating functions to deduce the case $(d, r, q)=(1,-1,1)$. At the end of Section 2 , we demonstrate that this algebraic argument can be replaced (and naturally generalized) by a combinatorial observation on abaci.

Section 3 is concerned with enumerating $s$-core abaci of bounded height. In particular, by looking at $s$-core abaci of bounded height, we provide a proof of Theorem 2 . We include applications of our approach in Section 4 by determining the maximum number of parts of certain core partitions and by enumerating core partitions with maximal initial gaps. As another application, we indicate in Section 5 how to determine the average number of parts (and, likewise, higher moments) of the core partitions studied here.

\section{$2 \quad$ Preliminaries and $s$-core abaci}

In this section, we introduce abaci and discuss their relation to core partitions. It is then shown in the next section that Theorem 2 on enumerating $(s, m s+r)$-core partitions is an instance of, and more naturally expressed as, a more general result on $s$-core abaci of bounded height.

An $s$-abacus is an array consisting of $s$ columns, labelled $0,1,2, \ldots, s-1$, and some number of rows, labelled $0,1,2, \ldots$, where each entry is either occupied by a bead or unoccupied (a spacer). The entry in row $i$ and column $j$ is said to be in position $i s+j$. 
Placing a set of nonnegative integers $\left\{a_{1}, a_{2}, \ldots\right\}$ on an $s$-abacus means to construct the $s$-abacus with beads precisely in the positions $a_{1}, a_{2}, \ldots$ The $s$-abacus corresponding to a partition $\lambda$ is obtained by placing the set of its first column hook lengths on an $s$-abacus. Consequently, the number of parts of $\lambda$ equals the number of beads of the corresponding abacus $A$, and the size of each part equals the number of spacers preceding the corresponding bead in $A$. We write $n(A)$ for the number of beads in $A$. We say that an $s$-abacus $A$ has spacing $\geqslant d$ if it corresponds to a partition with parts that differ by at least $d$. Equivalently, the positions of beads in $A$ differ by more than $d$.

In the sequel, an $s$-core abacus refers to an $s$-abacus corresponding to an $s$-core partition. By [13, Lemma 7], which is equivalent to [19, Lemma 2.1], these are characterized as follows.

Lemma 3 ([13, Lemma 7]). An s-abacus corresponds to an s-core partition if and only if the first column is empty and no spacers occur below a bead.

Example 4. The partition $\lambda=(7,5,4,2,1)$ has first column hook lengths $\{1,3,6,8,11\}$. Its 5 -abacus and 7 -abacus are:

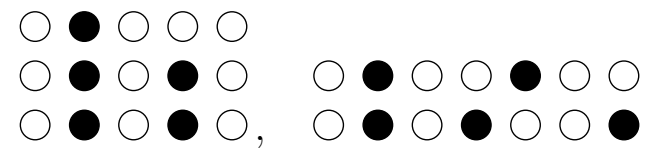

In light of Lemma 3, we see that $\lambda$ is not a 7 -core partition (because there is one bead with a spacer below it) but is a 5-core partition (because the first column is empty and no spacers occur below a bead). Because $\lambda$ has distinct parts, both abaci have spacing 1 .

Let $\mathcal{A}_{s, m}^{d}$ be the set of $s$-core abaci with (at most) $m$ rows and spacing $\geqslant d$ (throughout, $m \geqslant 1$ and $d \geqslant 1$ ). More generally, for integers $r$ (possibly negative), let $\mathcal{A}_{s, m, r}^{d}$ denote the set of $s$-core abaci with spacing $\geqslant d$ such that the maximum position is (strictly) less than $m s+r$. Note that, by definition, $\mathcal{A}_{s, m}^{d}=\mathcal{A}_{s, m, 0}^{d}$. (Since the first column of an $s$-core abacus is necessarily empty, we also have $\mathcal{A}_{s, m}^{d}=\mathcal{A}_{s, m, 1}^{d}$.) In the sequel, if $d=1$, we may omit $d$ in the notation. That is, $\mathcal{A}_{s, m}:=\mathcal{A}_{s, m}^{1}$ and $\mathcal{A}_{s, m, r}:=\mathcal{A}_{s, m, r}^{1}$.

Recall that $n(A)$ is the number of beads in the abacus $A$. Let

$$
\boldsymbol{A}_{s, m, r}^{d}(q)=\sum_{A \in \mathcal{A}_{s, m, r}^{d}} q^{n(A)}
$$

be the generating polynomial for the number of beads in the abaci in $\mathcal{A}_{s, m, r}^{d}$. As for $\mathcal{A}_{s, m, r}^{d}$, we drop $d$ and $r$ from the notation if $d=1$ or $r=0$, respectively. Note that $\boldsymbol{A}_{0, m, r}^{d}(q)=1$, representing the empty abacus. Since it is natural and convenient for certain results, we set $\boldsymbol{A}_{s, m, r}^{d}(q)=0$ for $s<0$.

Using the correspondence with abaci we can easily show the following result, which, in the case $d=1$, was proved in [18, Lemma 2.2] and [19, Lemma 3.2] and which is equivalent to [12, Lemma 2.5].

Lemma 5. If $1 \leqslant r \leqslant d$, then every $(s, s+r)$-core partition into d-distinct parts has maximum hook length $<s+r$. 
Proof. Let $A$ be the $s$-abacus of an $(s, s+r)$-core partition $\lambda$ into $d$-distinct parts. We need to show that all beads of $A$ (recall that these correspond to the first column hook lengths of $\lambda$ ) are in positions with labels $<s+r$. Assume for the sake of contradiction that $A$ has a bead in position $x \geqslant s+r$. By Lemma 3, because $\lambda$ is an $s$-core partition, there must also be a bead in position $x-s$. Likewise, because $\lambda$ is a $(s+r)$-core partition, there must be a bead in position $x-(s+r)$. However, $|(x-s)-(x-(s+r))|=r \leqslant d$, which contradicts the requirement that positions of beads in $A$ differ by more than $d$.

By construction, the abaci in $\mathcal{A}_{s, m, r}^{d}$ correspond to $s$-core partitions into $d$-distinct parts with largest hook $<m s+r$. Equivalently, these are $(s, m s+r, m s+r+1)$-core partitions into $d$-distinct parts (note that, by Lemma 5 applied with $d=r=1$, a $(t, t+1)$ core partition into distinct parts has largest hook less than $t$ ). If $r \leqslant d$, this simplifies as follows.

Lemma 6. If $1 \leqslant r \leqslant d$ or if $r=-1$, then the abaci in $\mathcal{A}_{s, m, r}^{d}$ are in $1-1$ correspondence with $(s, m s+r)$-core partitions into d-distinct parts.

Proof. We need to show that $(s, m s+r)$-core partitions into $d$-distinct parts have maximum hook length $<m s+r$. In the case $1 \leqslant r \leqslant d$, applying Lemma 5 with $m s$ in place of $s$, we find that every $(s, m s+r)$-core partition into $d$-distinct parts indeed has maximum hook length $<m s+r$.

Finally, consider the case $r<0$. Note that every $(s, m s+r)$-core partition is $(m s+$ $r, m s)$-core. Hence, if $d \geqslant|r|$, then Lemma 5 shows that every $(s, m s+r)$-core partition into $d$-distinct parts has maximum hook length $<m s$. If $r=-1$, it follows that the maximum hook length is $<m s-1$.

Example 7. Lemma 6 does not generally hold for other values of $r$. For instance, consider the case $r=-2$ and $(s, m)=(6,1)$. The partition $\lambda=(4,1)$ is $(s, m s+r)=(6,4)$-core and 3-distinct. However, its maximum hook length is $5 \nless m s+r=4$.

Lemma 8. Let $s>d+1$. Removing the last $d+1$ columns, not all empty, from an s-core abacus with spacing $\geqslant d$ results in an $(s-d-1)$-core abacus with spacing $\geqslant d$.

Proof. In light of Lemma 3, the result is clearly an $(s-d-1)$-core abacus. It remains to observe that the reduced abacus has spacing $\geqslant d$. For that, it suffices to check that, in the reduced abacus, each bead in the first row is followed by $d$ empty positions. Since the initial abacus had spacing $\geqslant d$, we need only consider the last such bead. In the initial abacus, this bead was followed by $d_{1} \geqslant d$ spacers, then a bead (in the last $d+1$ columns), followed by another $d_{2} \geqslant d$ spacers. After removal of the last $d+1$ columns, it is therefore followed by $d_{1}+d_{2}-d \geqslant d$ spacers.

Example 9. We note that this result is not true for removing the first $d+1$ columns. This is illustrated, for instance, with $d=2$, by the 5 -core abacus with beads in positions $1,4,9$. 
Nath and Sellers [13] prove Theorem 1 by a combinatorial argument for $(s, m s+1)$ core partitions. They then deduce the case of $(s, m s-1)$-core partitions by proving the $(q=1$ case of the) relation

$$
\boldsymbol{A}_{s, m,-1}(q)=\boldsymbol{A}_{s-1, m}(q)+\left(q+q^{2}+\ldots+q^{m-1}\right) \boldsymbol{A}_{s-2, m}(q)
$$

and using an algebraic argument based on generating functions. Note that, by Theorem 12 , the relation (4) is equivalent to

$$
\boldsymbol{A}_{s, m,-1}(q)=\boldsymbol{A}_{s, m}(q)-q^{m} \boldsymbol{A}_{s-2, m}(q) .
$$

Our next result provides a simple combinatorial proof of the following generalization of this identity. For the case $s=r$ in (5), it is understood that $\boldsymbol{A}_{s, m, r}^{d}(q)=0$ if $s<0$.

Lemma 10. Let $m, r \geqslant 1$. If $s \geqslant r$, then

$$
\boldsymbol{A}_{s, m}(q)=\boldsymbol{A}_{s, m,-r}(q)+q^{m} \sum_{k=1}^{r} \boldsymbol{A}_{s-k-1, m}(q) \boldsymbol{A}_{k-1, m-1}(q) .
$$

Proof. Suppose $s>r$. Observe that $\boldsymbol{A}_{s, m}(q)-\boldsymbol{A}_{s, m,-r}(q)$ consists of those $s$-core abaci with spacing $\geqslant 1$, which have $m$ rows and last bead in position $m s-k$ for $k=1,2, \ldots, r$. Fix one of these values for $k$, corresponding to one of the last $r$ columns. That column always contains $m$ beads, contributing $q^{m}$ to (5). The $k-1$ columns after that column form an abacus in $\mathcal{A}_{k-1, m-1}$, while the first $s-k-1$ columns form an abacus in $\mathcal{A}_{s-k-1, m}$. The one remaining column is the empty column preceding the column with $m$ beads.

If $s=r$, the same argument still applies but $k=r$ is not possible. Since $\boldsymbol{A}_{-1, m}(q)=0$, the summand corresponding to $k=r$ is zero, so that equation (5) still holds.

We conclude this section with some observations on abaci that should make the proof of Theorem 12 easier to follow.

Example 11. Consider an abacus in $\mathcal{A}_{s, m, r}^{d}$. Since, by definition, the maximum position is less than $m s+r$, such an abacus cannot have more than $m+\lceil r / s\rceil$ many rows. However, the number of rows can exceed $m+1$ when $r>s$.

On the other hand, assume as in Theorem 12 that $s>r \geqslant 0$ and $s>d+1$. Let $A$ be an abacus in $\mathcal{A}_{s-d-1, m, r}^{d}$. We claim that $A$ has at most $m+2$ rows and that the $(m+2)$ nd row contains at most one bead. To see this, note that the maximum position in such an abacus is less than

$$
m(s-d-1)+r<(m+1)(s-d-1)+d+1,
$$

which implies that, if there is a bead in the $(m+2)$ nd row (that is, a bead in position $(m+1)(s-d-1)+j$ for $j=1,2 \ldots, s-d-2)$, then there is no room for another bead in that row (because the abacus has spacing $\geqslant d$ ). Further note that, in order to have beads beyond the first row, we need to have $s-d-1>d+1$. Taking that into account, we see that it is impossible to have a bead in the $(m+3)$ rd row (because $(m+2)(s-d-1)+j>(m+1)(s-d-1)+d+1+j$ exceeds the maximum possible position). 


\section{$3 s$-core abaci of bounded height}

As introduced in Section $2, \mathcal{A}_{s, m, r}^{d}$ is the set of $s$-core abaci with spacing $\geqslant d$ such that the maximum position is less than $m s+r$. Recall that $n(A)$ is the number of beads in $A$.

As a consequence of Lemma 6 , if $1 \leqslant r \leqslant d$ or if $r=-1$, then

$$
C_{s, m, r}^{d}(q)=\boldsymbol{A}_{s, m, r}^{d}(q)
$$

where $\boldsymbol{C}_{s, m, r}^{d}(q)$, defined in (2), is the generating polynomial for the number of parts of $(s, m s+r)$-core partitions into $d$-distinct parts. In particular, Theorem 2 on core partitions is contained in the following result on abaci.

Theorem 12. Let $d, m \geqslant 1, r \geqslant 0$, and write $f_{s}(q)=\boldsymbol{A}_{s, m, r}^{d}(q)$. If $s>d+1$ and $s>r$, then

$$
f_{s}(q)=f_{s-1}(q)+\left(q+q^{2}+\ldots+q^{m}\right) f_{s-d-1}(q) .
$$

We note that, in the case $d=1$, the condition $s>d+1$ may be dropped, because (7) trivially holds for $s=1$ and $s=2$ (with the understanding that $f_{s}(q)=0$ if $s<0$ ).

Proof. By definition, $f_{s}(q)$ is the generating polynomial for $s$-core abaci with spacing $\geqslant d$, which consist of $m+1$ rows such that the top row has beads only in the first $r$ positions. Let $g$ be the size of the gap between the last bead in the first row and the first bead in the second row; or $g=\infty$ if there are no beads in the second row. By assumption, $g \geqslant d$.

There are two disjoint possibilities for such abaci, depending on whether $g>d$ and the last column is empty (case (a)), or not (case (b)).

(a) Suppose that $g>d$ and that the last column is empty. Since $r \leqslant s-1$, by removing the last column, we see that these abaci are in bijective correspondence with abaci in $\mathcal{A}_{s-1, m, r}^{d}$. The generating polynomial for the latter is $f_{s-1}(q)$.

(b) Suppose that $g=d$ or that the last column is non-empty. In that case, exactly one of the last $d+1$ columns contains beads. We must again consider two cases based on whether the last bead (that is, the bead in the highest position) is located in the last $d+1$ columns of the $(m+1)$ st row (case (b)ii) or not (case (b)i). (We note that, if $s>r+d+1$, then we are necessarily in case (b)i.)

(i) Suppose the last bead is not located in the last $d+1$ columns of the $(m+$ 1 )st row (that is, its position is $<(m+1) s-d-1)$. Consider the abaci obtained by removing the last $d+1$ columns, exactly one of which contains beads $\left(1,2, \ldots, m\right.$ many). By Lemma 8 , the resulting abaci are in $\mathcal{A}_{s-d-1, m, r}^{d}$ with no bead in the $(m+2)$ nd row.

To see that this correspondence is $m$ to 1 , consider an abacus $A$ in $\mathcal{A}_{s-d-1, m, r}^{d}$ with no bead in the $m+2$ nd row. We need to show that there are exactly $m$ ways in which $A$ could have been obtained from a case (b)i abacus $B \in \mathcal{A}_{s, m, r}^{d}$ by deleting the last $d+1$ columns. Let $h \geqslant 1$ be the number of empty positions at the beginning of the second row of $A$. If $h \leqslant d$, then the last $d-h$ columns 
of $B$ must have been empty and the column before that non-empty (with $m$ choices for the number of beads). If $h>d$, then the last column of $B$ must have been non-empty (again, with $m$ choices for the number of beads). In either case, the remaining of the last $d+1$ columns of $B$ must be empty. By construction, each of the $m$ possibilities for $B$ indeed are case (b)i abaci from $\mathcal{A}_{s, m, r}^{d}$.

(ii) We now are left with abaci in which the last bead is located in the last $d+1$ columns of the $(m+1)$ st row. Equivalently, the unique non-empty column among the last $d+1$ has $m+1$ beads. Since $s>r$, this cannot be the last column. Consequently, $g=d$. In particular, there is a bead in the second row of the first non-empty column. Increase the number of beads in that first non-empty column to $m+2$ (thereby adding $1,2, \ldots, m$ beads), and remove the last $d+1$ columns (thereby removing $m+1$ beads). This process removes $1,2, \ldots, m$ beads. By Lemma 8 , the resulting abaci are in $\mathcal{A}_{s-d-1, m, r}^{d}$ with a bead in the $(m+2)$ nd row.

To see that this correspondence is $m$ to 1 , consider an abacus $A$ in $\mathcal{A}_{s-d-1, m, r}^{d}$ with a bead in the $(m+2)$ nd row. (By Example 11, abaci in $\mathcal{A}_{s-d-1, m, r}^{d}$ have at most $m+2$ rows and at most one bead in the $(m+2)$ nd row.) We need to show that there are exactly $m$ ways in which $A$ could have been obtained from a case (b)ii abacus $B \in \mathcal{A}_{s, m, r}^{d}$. As in case (b)i, let $h \geqslant 1$ be the number of empty positions at the beginning of the second row of $A$. Note that column $h+1$ of $A$ contains $m+2$ beads. We necessarily have $h \leqslant d$, so that the last $d-h$ columns of $B$ must have been empty and the column before that must have contained $m+1$ beads. In order to obtain $A$ from $B$, column $h+1$ of $B$ must have contained $2,3, \ldots, m+1$ beads, for a total of $m$ choices for $B$.

Example 13. To help illustrate the proof of Theorem 12 in the case $d=2, s=6, m=2$ and $r=5$, consider the following three abaci which are members of the set $\mathcal{A}_{6,2,5}^{2}$.

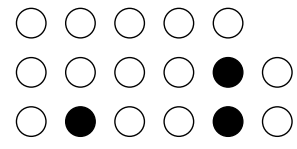

A

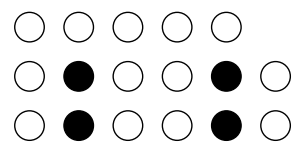

$B$

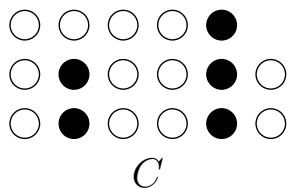

C

Proceeding as in the proof of Theorem 12 , these abaci $A, B, C$ get mapped to the following three abaci $A^{\prime}, B^{\prime}, C^{\prime}$.

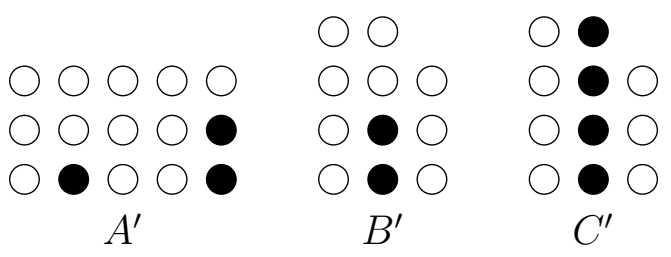

Indeed, for abacus $A$, we have $g=5>2=d$, so, following case (a), we remove the last column to obtain the abacus $A^{\prime}$ in $\mathcal{A}_{5,2,5}^{2}$. 
For abacus $B$, we have $g=2=d$ and the final bead is not in the last $d+1=3$ columns of the third row, so we follow the map described in case (b)i and remove the final $d+1=3$ columns to obtain the abacus $B^{\prime}$ in $\mathcal{A}_{3,2,5}^{2}$. Note that the abacus $B^{\prime}$ would have also been the result of applying our map to the abacus obtained by removing the second bead from the fourth column of abacus $B$.

Finally, for abacus $C$, we have $g=2=d$ and the final bead is in the last $d+1=3$ columns of the third row. Thus, following the map described in case (b)ii, we obtain the abacus $C^{\prime}$ in $\mathcal{A}_{3,2,5}^{2}$, removing one bead in the process.

Theorem 12 has the following analog for abaci in $\mathcal{A}_{s, m, r}^{d}$ where $r$ is negative. Because of Lemma 6, the case $r=-1$ is of particular interest, as those abaci correspond to $(s, m s-1)$-core partitions into $d$-distinct parts. As such, Theorem 14 (together with Lemma 15) proves the part of Theorem 2 concerned with these partitions.

Theorem 14. Let $d, m \geqslant 1, r>0$, and write $f_{s}(q)=\boldsymbol{A}_{s, m,-r}^{d}(q)$. If $s>2 d+r$, then

$$
f_{s}(q)=f_{s-1}(q)+\left(q+q^{2}+\ldots+q^{m}\right) f_{s-d-1}(q) .
$$

It appears that the bound $s>2 d+r$ is sharp for large $r$, specifically $r>d+1$, and $d \geqslant 2$. For small $r$, the bound can be improved to $s>2 d+1$. We give a direct combinatorial proof, in the spirit of Theorem 12, of this fact in Theorem 16. Based on numerical evidence, the optimal lower bounds on $s$ appear to be

$$
s>2 d+ \begin{cases}r, & \text { if } r>d+1 \text { and } d \geqslant 2, \\ 1, & \text { if } r \leqslant d+1 \text { and } d \geqslant 2, \\ r-1, & \text { if } d=1\end{cases}
$$

We prove the remaining case $d=1$ in Lemma 17 .

Proof of Theorem 14. Let us prove the claim that (8) holds for all $s>2 d+r$. Fix $r>1$ and suppose, for the purposes of induction, that this claim is true for $r$ replaced with $1,2, \ldots, r-1$. In the following, we are going to show that the claim holds for $r$ as well. [Along the way, we also show that (8) holds for $r=1$ and all $s>2 d+2$. Comments and adjustments for that case are included in square brackets. Since (8) is proved for $r=1$ and $s=2 d+2$ in Lemma 15 below, this completes the proof of Theorem 14.]

Suppose that $s>2 d+r$ [if $r=1$, then $s>2 d+2$ ]. In the following, let $t$ be such that $t \geqslant s-d-1$ (we intend to rewrite each of the terms $f_{t}(q)$ in (8)). Note that the condition $s>2 d+r$ translates into $t>d+r-1$ [while, for $r=1$, the condition $s>2 d+2$ translates into $t>d+1$.

By construction, for any integer $t \geqslant 0$, we have

$$
\boldsymbol{A}_{t, m,-r}^{d}(q)=\boldsymbol{A}_{t, m-1, t-r}^{d}(q) .
$$

Since $t \geqslant r$ and $t>d+1$, we may apply Theorem 12 to the right-hand side of (9) to obtain

$$
\begin{aligned}
\boldsymbol{A}_{t, m,-r}^{d}(q) & =\boldsymbol{A}_{t-1, m-1, t-r}^{d}(q)+\left(q+q^{2}+\ldots+q^{m-1}\right) \boldsymbol{A}_{t-d-1, m-1, t-r}^{d}(q) \\
& =\boldsymbol{A}_{t-1, m, 1-r}^{d}(q)+\left(q+q^{2}+\ldots+q^{m-1}\right) \boldsymbol{A}_{t-d-1, m, d+1-r}^{d}(q) .
\end{aligned}
$$


For the second equality, we applied (9), in reverse, to both summands. On the other hand, we have

$$
\boldsymbol{A}_{t, m, d+1-r}^{d}(q)=\boldsymbol{A}_{t-1, m, d+1-r}^{d}(q)+\left(q+q^{2}+\ldots+q^{m}\right) \boldsymbol{A}_{t-d-1, m, d+1-r}^{d}(q),
$$

as a consequence of Theorem 12, if $d+1-r \geqslant 0$, or the induction hypothesis (since, if $d+1-r<0$, then the condition $t>2 d+(r-d-1)=d+r-1$ is satisfied). [If $r=1$, then no induction hypothesis is required because we will always be in the case $d+1-r \geqslant 0$.]

Comparing the previous two equations, we conclude that, for $t \geqslant s-d-1$,

$$
\begin{aligned}
\boldsymbol{A}_{t, m,-r}^{d}(q) & =\boldsymbol{A}_{t-1, m, 1-r}^{d}(q)-q^{m} \boldsymbol{A}_{t-d-1, m, d+1-r}^{d}(q) \\
& +\boldsymbol{A}_{t, m, d+1-r}^{d}(q)-\boldsymbol{A}_{t-1, m, d+1-r}^{d}(q) .
\end{aligned}
$$

Let $g_{t}(q)$ be any one of the four terms on the right-hand side of (10). As above, after checking that the conditions are satisfied, it follows from either Theorem 12 or the induction hypothesis that, for the special case $t=s$,

$$
g_{s}(q)=g_{s-1}(q)+\left(q+q^{2}+\ldots+q^{m}\right) g_{s-d-1}(q),
$$

which then shows that the same relation holds for $f_{s}(q)=\boldsymbol{A}_{s, m,-r}^{d}(q)$, thus establishing the claimed relation (8). Let us illustrate the details of checking (11) at the example of $g_{s}(q)=\boldsymbol{A}_{s-d-1, m, d+1-r}^{d}(q)$, which is the most restrictive of the four cases. In that case, if $d+1-r \geqslant 0$, then Theorem 12 applies to show (11) because the condition $s-d-1>d+1$, that is $s>2 d+2$, follows from the assumption that $s>2 d+r$ [whereas, in the case $r=1$, we are assuming $s>2 d+2$ to begin with]. On the other hand, if $d+1-r<0$ [this does not happen when $r=1$ ], then the induction hypothesis applies to show (11) because the assumed condition $s>2 d+r$ implies the necessary condition $s-d-1>2 d+(r-d-1)$.

We now complete the proof of Theorem 14 by showing that, in the case $r=1,(8)$ holds for $s=2 d+2$.

Lemma 15. Let $d, m \geqslant 1$, and write $f_{s}(q)=\boldsymbol{A}_{s, m,-1}^{d}(q)$. If $s=2 d+2$, then (8) holds.

Proof. We need to show that

$$
\boldsymbol{A}_{2 d+2, m,-1}^{d}(q)=\boldsymbol{A}_{2 d+1, m,-1}^{d}(q)+\left(q+q^{2}+\ldots+q^{m}\right) \boldsymbol{A}_{d+1, m,-1}^{d}(q) .
$$

Because each abacus in $\mathcal{A}_{d+1, m,-1}^{d}$ has at most one column of beads,

$$
\begin{aligned}
\boldsymbol{A}_{d+1, m,-1}^{d}(q) & =1+\left(q+q^{2}+\ldots+q^{m-1}\right)+(d-1)\left(q+q^{2}+\ldots+q^{m}\right) \\
& =(1+(d-1) q)\left(1+q+\ldots+q^{m-1}\right) .
\end{aligned}
$$

On the other hand, abaci in $\mathcal{A}_{2 d+2, m,-1}^{d}$ and $\mathcal{A}_{2 d+1, m,-1}^{d}$ have at most two columns of beads. Appending an empty column to each abacus in $\mathcal{A}_{2 d+1, m,-1}^{d}$ yields an abacus in $\mathcal{A}_{2 d+2, m,-1}^{d}$. The abaci in $\mathcal{A}_{2 d+2, m,-1}^{d}$ not obtained in this fashion fall into three groups: 
(i) Those for which the last column is not empty (and hence contains $1,2, \ldots, m-1$ many beads). The generating polynomial for these abaci is

$$
\left(q+q^{2}+\ldots+q^{m-1}\right)\left(1+\left(q+q^{2}+\ldots+q^{m}\right)+(d-1) q\right)
$$

based on whether there is no second column of beads (contributing the 1 in the second factor), or there is a column of beads including position $d$ (contributing $q+q^{2}+\ldots+q^{m}$ ), or there is a single bead in positions $1,2, \ldots, d-1$ (contributing $(d-1) q)$.

(ii) Those for which the last column is empty and there is a gap of exactly $d$ spaces between the last bead in the first row and the first bead in the second row. Since there are $d-1$ possibilities for the location of the first column of beads (which contains $2,3, \ldots, m$ many beads), each of which determines the location of the second column (containing $1,2, \ldots, m$ beads), the generating polynomial for these abaci is

$$
(d-1)\left(q^{2}+q^{3}+\ldots+q^{m}\right)\left(q+q^{2}+\ldots+q^{m}\right) .
$$

(iii) Those for which the second to last column contains $m$ beads and, to avoid double counting, there are more than $d$ spaces following the last bead in the first row. The generating polynomial for these abaci is

$$
q^{m}(1+(d-1) q)
$$

because, in addition to the column of $m$ beads, there can only be a single bead in positions $1,2, \ldots, d-1$.

Adding these three generating polynomials, we have shown that

$$
\boldsymbol{A}_{2 d+2, m,-1}^{d}(q)-\boldsymbol{A}_{2 d+1, m,-1}^{d}(q)=q(1+(d-1) q)\left(1+q+\ldots+q^{m-1}\right)^{2} .
$$

Together with (13), this proves (12).

The bound $s>2 d+r$ in Theorem 14 can be improved to $s>2 d+1$ in the case of small $r$, specifically $r \leqslant d+1$. We give a direct combinatorial proof for that improved bound, which empirically is best possible.

Theorem 16. Let $m \geqslant 1,1 \leqslant r \leqslant d+1$, and write $f_{s}(q)=\boldsymbol{A}_{s, m,-r}^{d}(q)$. If $s>2 d+1$, then

$$
f_{s}(q)=f_{s-1}(q)+\left(q+q^{2}+\ldots+q^{m}\right) f_{s-d-1}(q) .
$$

Proof. Our proof is a variation of the proof of Theorem 12. By definition, $f_{s}(q)$ is the generating polynomial for $s$-core abaci with spacing $\geqslant d$ which consist of $m$ rows such that the top row has no beads in the last $r$ positions. As in the proof of Theorem 12, let $g$ be the size of the gap between the last bead in the first row and the first bead in the second row. There are two disjoint possibilities for such abaci. 
(a) Suppose that $g>d$ with the last column empty and no bead in the final allowed position (that is, no bead in position $m s-r-1$ ). By removing the last column, we see that these abaci are in bijective correspondence with abaci in $\mathcal{A}_{s-1, m, r}^{d}$.

(b) Suppose the conditions of (a) are not satisfied. We distinguish the following cases.

(i) Suppose that the last $d+1$ columns are empty. This can only happen if $r=d+1$ and if there is a bead in the highest allowed position (that is, column $s-r-1$ has $m$ beads). We then remove the last $d+1$ columns, as well as the $m$ beads from column $s-r-1$, and obtain an abacus in $\mathcal{A}_{s-d-1, m,-r}^{d}$.

(ii) Otherwise, there is precisely one non-empty column among the last $d+1$ columns. Suppose (additionally) that one of the $r$ columns $s-d-r, \ldots, s-$ $d-2, s-d-1$, say column $j$, contains $m$ beads (equivalently, removing the last $d+1$ columns does not result in an abacus in $\left.\mathcal{A}_{s-d-1, m,-r}^{d}\right)$. In that case, the one non-empty column among the last $d+1$ must be one of the last $r$ and, as such, contains $b<m$ beads. We then remove the last $d+1$ columns and reduce the number of beads in column $j$ by $m-b$. The resulting abacus is in $\mathcal{A}_{s-d-1, m,-r}^{d}$ and has precisely $m$ fewer beads.

(iii) Otherwise, we remove the last $d+1$ columns and, by Lemma 8 , obtain an abacus in $\mathcal{A}_{s-d-1, m,-r}^{d}$.

Fix an abacus $A \in \mathcal{A}_{s-d-1, m,-r}^{d}$. To complete the proof, we need to show that $A$ is obtained through the described process from $m$ case (b) abaci $B \in \mathcal{A}_{s, m,-r}^{d}$, with $1,2, \ldots, m$ additional beads. Let $h \geqslant 1$ be the number of empty positions at the beginning of the second row of $A$. If $h \leqslant d$, then let $k=s-(d-h)$. Otherwise, let $k=s$. We can construct $B$ from $A$ by adding $d+1$ empty columns, and then filling column $k$ with $b$ beads, where $1 \leqslant b<m$. (Note that, if $h \leqslant d$, then $g=d$ for $B$, while otherwise $B$ has a non-empty last column.) It remains to similarly construct an abacus $B$ from $A$ by adding $d+1$ columns and $m$ beads. For that, we distinguish three cases:

- If column $k$ is not among the last $r$ columns of $B$, then it can also be filled with $m$ beads.

- Otherwise, if one of the last $r$ columns of $A$ contains $c \geqslant 1$ beads, then we add $m-c$ beads to the corresponding column of $B$ and $c$ beads to column $k$ of $B$.

- Otherwise, we add $m$ beads to column $s-r-1$ of $B$.

In each case, we check that abacus $B$ corresponds to case (b) and results in abacus $A$ by the process described for case (b).

We further provide a short proof that Theorem 14, in the case $d=1$, continues to hold for all $s>r+1$, which empirically is best possible.

Lemma 17. Let $m, r \geqslant 1$, and write $f_{s}(q)=\boldsymbol{A}_{s, m,-r}(q)$. If $s>r+1$, then (8) holds. 
Proof. By Lemma 10, for $s \geqslant r$,

$$
\boldsymbol{A}_{s, m,-r}(q)=\boldsymbol{A}_{s, m}(q)-q^{m} \sum_{k=1}^{r} \boldsymbol{A}_{s-k-1, m}(q) \boldsymbol{A}_{k-1, m-1}(q) .
$$

Let $g_{s}(q)$ be any one of the terms $\boldsymbol{A}_{s, m}(q)$ or $\boldsymbol{A}_{s-k-1, m}(q)$, for $k=1,2, \ldots, r$, on the right-hand side. To prove our claim, it suffices to show that, for $s \geqslant r+2$,

$$
g_{s}(q)=g_{s-1}(q)+\left(q+q^{2}+\ldots+q^{m}\right) g_{s-2}(q) .
$$

This follows from Theorem 12 with $r=0$ (recalling that, for $d=1$, the condition $s>d+1$ may be dropped).

We close this section by showing that Theorem 12 can be used to compute $\boldsymbol{A}_{s, m, r}^{d}(q)$ for any choice of the parameters, that is for any $s, m, d \geqslant 1$ and any integers $r$. First, note that, if $0 \leqslant m s+r<s$, then $\boldsymbol{A}_{s, m, r}^{d}(q)=\boldsymbol{A}_{m s+r, 1,0}^{d}(q)$ (if $m s+r<0$, then $\boldsymbol{A}_{s, m, r}^{d}(q)=1$ ). Second, if $m s+r \geqslant s$, we can write $m s+r=m^{\prime} s+r^{\prime}$ where $m^{\prime} \geqslant 1$ and $0 \leqslant r^{\prime}<s$. By definition,

$$
\boldsymbol{A}_{s, m, r}^{d}(q)=\boldsymbol{A}_{s, m^{\prime}, r^{\prime}}^{d}(q) .
$$

These observations reduce the general case to the case $s>r \geqslant 0$. In light of Theorem 12 , the following initial conditions therefore suffice to recursively determine $\boldsymbol{A}_{s, m, r}^{d}(q)$ for any choice of the parameters. In the following, we use the notation $x^{+}=\max (0, x)$.

Lemma 18. Let $d, m \geqslant 1$ and $r$ be any integer. For $s \in\{1,2, \ldots, d\}$,

$$
\boldsymbol{A}_{s, m, r}^{d}(q)=1+\min (s-1, m s+r-1)^{+} q .
$$

For the case $s=d+1$, write $r=s r^{\prime}+r_{0}$ with $0 \leqslant r_{0} \leqslant d$. If $m+r^{\prime}<0$, then $\boldsymbol{A}_{d+1, m, r}^{d}(q)=1$. If $m+r^{\prime} \geqslant 0$, then

$$
\boldsymbol{A}_{d+1, m, r}^{d}(q)=1+d\left(q+q^{2}+\ldots+q^{m+r^{\prime}}\right)+\left(r_{0}-1\right)^{+} q^{m+r^{\prime}+1} .
$$

Proof. The case $s \in\{1,2, \ldots, d\}$ is an immediate consequence of the fact that the corresponding abaci can have at most one bead.

In the case $s=d+1$, we can have a full column of beads in any of the $s-1=$ $d$ columns after the first (and there can be no bead in a second column). Note that $\mathcal{A}_{d+1, m, r}^{d}=\mathcal{A}_{d+1, m+r^{\prime}, r_{0}}^{d}$. Since the case $m+r^{\prime}<0$ is trivial, suppose $m+r^{\prime} \geqslant 0$. Each of the last $d$ columns can accomodate $m+r^{\prime}$ many beads. Additionally, any of the $r_{0}-1$ columns $1,2,3, \ldots, r_{0}-1$ can accomodate an additional bead in the $\left(m+r^{\prime}+1\right)$ st row.

Example 19. In the case $d=1$, we find that $\boldsymbol{A}_{1, m, r}(q)=1$ and

$$
\boldsymbol{A}_{2, m, r}(q)=1+q+q^{2}+\ldots+q^{m+\lfloor r / 2\rfloor} .
$$

In the cases $r= \pm 1, q=1$, these specialize to the initial conditions in Theorem 1 . 
Example 20. Recall from (6) that, in the case $(d, r)=(1,-1)$, we have $\boldsymbol{A}_{s, m, r}(q)=$ $\boldsymbol{C}_{s, m, r}(q)$. For instance, for $(s, m)=(4,3)$ we have $m s-1=11$ and the $(4,11)$-core partitions into distinct parts are
$\emptyset, \quad(1)$
(2),
(3), $\quad(2,1)$,
$(4,1)$,
$(5,2), \quad(6,3)$,
$(3,2,1)$,
$(5,2,1)$,
$(7,4,1)$
$(8,5,2)$,
$(4,3,2,1), \quad(6,3,2,1)$,
$(5,4,3,2,1)$.

There are $\boldsymbol{C}_{4,3,-1}(1)=15$ such partitions and the generating polynomial for the number of parts in these partitions is

$$
\boldsymbol{C}_{4,3,-1}(q)=1+3 q+4 q^{2}+4 q^{3}+2 q^{4}+q^{5}=\left(1+q+q^{2}\right)\left(1+2 q+q^{2}+q^{3}\right) .
$$

We note that such a factorization always exists for the polynomials $\boldsymbol{C}_{s, m,-1}(q)$. Indeed, for $s \geqslant 2$, we claim that $\boldsymbol{C}_{s, m,-1}(q)$ is divisible by $1+q+q^{2}+\ldots+q^{m-1}$. This is true for $\boldsymbol{C}_{2, m,-1}(q)$ by (15) and the claim follows inductively from Theorem 2.

Example 21. Continuing the previous example in the special case $m=2$, we conclude from Theorem 2 that

$$
\boldsymbol{C}_{s, 2,-1}(q)=(1+q)^{s-1} .
$$

In particular, the number of $(s, 2 s-1)$-core partitions into $k$ distinct parts is $\left(\begin{array}{c}s-1 \\ k\end{array}\right)$. Less generally, as observed in [18], there are $2^{s-1}$ many $(s, 2 s-1)$-core partitions into distinct parts.

Remark 22. Example 21 suggests that there is a natural correspondence of compositions of $s$ and $(s, 2 s-1)$-core partitions into distinct parts. Indeed, let us briefly describe a bijection between compositions $\mu$ of $s$ into $k$ parts and $(s, 2 s-1)$-core partitions $\lambda$ into $k-1$ distinct parts. Given a partition $\lambda$, the corresponding composition $\mu=\left(\mu_{1}, \mu_{2}, \ldots, \mu_{k}\right)$ of $s$ is defined as follows. Consider the $s$-abacus of $\lambda$, and let $\mu_{1}>0$ be the number of empty columns before the first bead. Then we define $\mu_{j+1}$, for $j=1,2, \ldots, k-1$, depending on the location of the $j$ th bead in the abacus.

(a) If the $j$ th bead is the only bead in its columns, then $\mu_{j+1}$ is 1 more than the number of empty columns following that bead.

(b) If the $j$ th bead is the first of two beads in its columns, then $\mu_{j+1}=1$.

(c) If the $j$ th bead is the second of two beads in its columns, then $\mu_{j+1}$ is the number of empty columns following that bead.

Note that the size $\mu_{1}+\ldots+\mu_{k}$ of the resulting composition $\mu$ is exactly $s$, the number of columns in the $s$-abacus of $\lambda$. We leave it to the interested reader to confirm that this correspondence is indeed a bijection. 


\section{Maximum number of parts and partitions with maximal ini- tial gaps}

The correspondence with abaci makes certain properties of $s$-core abaci conveniently accessible. For instance, we can readily deduce the maximum number of parts of the $s$-core partitions featured in Theorem 2.

Lemma 23. Let $M_{s, m, r}^{d}$ be the maximum number of parts of $(s, m s+r)$-core partitions into $d$-distinct parts. Let $d, m \geqslant 1$. If $1 \leqslant r \leqslant d$ and $s>1$, then

$$
M_{s, m, r}^{d}=\left\lfloor\frac{s}{d+1}\right\rfloor m+ \begin{cases}0, & \text { if } r=1 \text { and } s \equiv 0,1 \quad(\bmod d+1), \\ 1, & \text { otherwise. }\end{cases}
$$

Likewise, if $r=-1$ and $s>2$, then

$$
M_{s, m,-1}^{d}=\left\lfloor\frac{s}{d+1}\right\rfloor m+ \begin{cases}-1, & \text { if } d=1 \text { and } s \equiv 0 \quad(\bmod 2), \\ 0, & \text { otherwise, } \text { if } s \equiv 0,1,2 \quad(\bmod d+1) \\ 1, & \text { otherwise }\end{cases}
$$

Proof. Observe that, by definition, $M_{s, m, r}^{d}=\operatorname{deg}\left(\boldsymbol{C}_{s, m, r}^{d}(q)\right)$. The claim could therefore be deduced inductively from Theorem 2 together with the initial conditions in Lemma 18.

Instead, in Lemma 24, we provide a combinatorial argument that explicitly constructs a partition with the maximum number of parts (or, equivalently, the corresponding $s$-core abacus). The second part of our claim, the case $r=-1$, follows from using $M_{s, m,-1}^{d}=$ $M_{s, m-1, s-1}^{d}$ in Lemma 24 .

Note that, by Lemma 6, the definition of $M_{s, m, r}^{d}$ in Lemma 24 agrees with the definition in Lemma 23 if $1 \leqslant r \leqslant d$ or if $r=-1$.

Lemma 24. Let $M_{s, m, r}^{d}$ be the maximum number of beads of an abacus in $\mathcal{A}_{s, m, r}^{d}$. Let $d, m \geqslant 1$. If $s \geqslant r=1$, then (17) holds. If $s \geqslant r>1$, then

$$
M_{s, m, r}^{d}=\left\lfloor\frac{s}{d+1}\right\rfloor m+\left\lfloor\frac{r-2}{d+1}\right\rfloor+1 .
$$

Proof. Since the maximum number of beads is always achieved by an abacus with a bead in position 1, we assume in the sequel that there is a bead in position 1. Since there are $s-2$ further positions in the first row, the maximum number of beads in the first row of an abacus in $\mathcal{A}_{s, m, r}^{d}$ is

$$
1+\left\lfloor\frac{s-2}{d+1}\right\rfloor
$$

Observe that each of the $m-1$ subsequent rows can be filled with up to $\lfloor s /(d+1)\rfloor$ many beads. Note that (19) either equals $\lfloor s /(d+1)\rfloor$ or exceeds it by 1 . The former case happens precisely if $s \equiv 0,1$ modulo $d+1$. This proves (17) in the case $r=1$.

In the case that (19) equals $\lfloor s /(d+1)\rfloor$, the $(m+1)$ st row may be filled with 1 bead in position $m s+1$ plus an additional $\lfloor(r-2) /(d+1)\rfloor$ beads. In the case that (19) exceeds 
$\lfloor s /(d+1)\rfloor$ by 1 (then the second column is empty except for a bead in the first row), the $(m+1)$ st row may be filled with $\lfloor(r-2) /(d+1)\rfloor$ beads. In either case, we find that the maximum total number of beads is (18).

For comparison, let us consider $M_{s, t}$, the maximum number of parts of an $(s, t)$-core partition (without any restriction on its parts). Equivalently, $M_{s, t}$ is the largest part of an $(s, t)$-core partition. The following is a corollary of Sylvester's theorem on the Frobenius problem; we refer to [7, Chapter 1] for a beautiful exposition.

Lemma 25. For coprime $s, t>1$, we have $M_{s, t}=\frac{1}{2}(s-1)(t-1)$.

Proof. As discussed in Section 2, we can identify partitions $\lambda$ with the set $A(\lambda)$ of their first column hook lengths (these are precisely the positions of beads in the corresponding abaci). Note that $|A(\lambda)|$ is the number of parts of $\lambda$.

By Lemma 3, $\lambda$ is $s$-core if and only if $s \leqslant h \in A(\lambda)$ implies that $h-s \in A(\lambda)$. It follows that, if $\lambda$ is $\left(s_{1}, \ldots, s_{n}\right)$-core, then $A(\lambda)$ is a subset of the set $F\left(s_{1}, \ldots, s_{n}\right)$ of positive integers which cannot be written as a nonnegative linear combination of $s_{1}, \ldots, s_{n}$. Moreover, the set $F\left(s_{1}, \ldots, s_{n}\right)$ itself corresponds to a $\left(s_{1}, \ldots, s_{n}\right)$-core partition. It was shown by Sylvester [7, Theorem 1.3] that

$$
|F(s, t)|=\frac{(s-1)(t-1)}{2},
$$

proving our claim.

It is considerably more difficult to obtain the maximum size of core partitions. In this direction, we only mention the following well-known result of Olsson and Stanton [14].

Theorem 26. Suppose s and $t$ are coprime. The maximum size of an $(s, t)$-core partition is

$$
\frac{\left(s^{2}-1\right)\left(t^{2}-1\right)}{24} \text {. }
$$

Recall that $\mathcal{C}_{s, m, r}^{d}$ is the set of $(s, m s+r)$-core partitions into $d$-distinct parts. The enumerations in Theorem 2 are based on a recursive description in $s$ with $m$ (as well as $r$ and $d$ ) fixed. Note that $\mathcal{C}_{s, m, r} \subseteq \mathcal{C}_{s, m+1, r}$. In the remainder of this section, we observe another way in which $\mathcal{C}_{s, m, r}$ is naturally embedded in $\mathcal{C}_{s, m+1, r}$.

We say that the initial gap of an integer partition $\left(\lambda_{1}, \lambda_{2}, \ldots\right)$, with $\lambda_{1} \geqslant \lambda_{2} \geqslant \ldots$, is the difference $\lambda_{1}-\lambda_{2}$ of the first and second part. If a partition $\lambda$ is $s$-core, then all the gaps between the parts of $\lambda$ are strictly less than $s$. We say that a $\left(s_{1}, s_{2}, \ldots\right)$-core partition $\lambda$ has maximal initial gap if $\lambda$ has initial gap $\min \left(s_{1}, s_{2}, \ldots\right)-1$.

Let $\mathcal{G}_{s, m, r}^{d}$ be the set of partitions $\lambda \in \mathcal{C}_{s, m, r}^{d}$ with maximal initial gap, together with the empty partition (in other words, $\mathcal{G}_{s, m, r}^{d}$ consists of those $\lambda \in \mathcal{C}_{s, m, r}^{d}$ such that, if $\lambda$ has a first part $\lambda_{1}$, then it has a second part $\lambda_{2}$ and $\left.\lambda_{1}-\lambda_{2}=s-1\right)$.

Lemma 27. Let $s, m \geqslant 1$. If $1 \leqslant r \leqslant d$ or $r=-1$, then there is a natural 1-1 correspondence between $(s, m s+r)$-core partitions into d-distinct parts with maximal initial gap and $(s,(m-1) s+r)$-core partitions into d-distinct parts 
Proof. The claimed bijective correspondence $\mathcal{G}_{s, m, r}^{d} \rightarrow \mathcal{C}_{s, m-1, r}^{d}$ is given by the map

$$
\left(\lambda_{1}, \lambda_{2}, \lambda_{3}, \ldots\right) \mapsto\left(\lambda_{2}, \lambda_{3}, \ldots\right),
$$

with the understanding that the empty partition is sent to itself.

Corollary 28. In particular, if $1 \leqslant r \leqslant d$ or $r=-1$, we get an enumeration of $(s, m s+r)$ core partitions into d-distinct parts with maximal initial gap from Theorem 2.

Example 29. The number of $(s, 3 s-1)$-core partitions into $k$ distinct parts with maximal initial gap is $\left(\begin{array}{c}s-1 \\ k-1\end{array}\right)$. This follows from the bijective correspondence between $\mathcal{G}_{s, 3,-1}^{1}$ and $\mathcal{C}_{s, 2,-1}^{1}$. Indeed, for the latter, as noted in (16), the generating polynomial for the number of parts of $(s, 2 s-1)$-core partitions into distinct parts is $\boldsymbol{C}_{s, 2,-1}(q)=(1+q)^{s-1}$. It was this example that initially motivated the present paper.

\section{Average number of parts}

In this section, we indicate that our results on the enumeration of certain $(s, t)$-core partitions can be used to obtain explicit formulas for the averge number of parts of these partitions.

Determining the average size of core partitions has received considerable attention in recent years. In particular, Johnson [11] proved the following result, which had been experimentally observed and conjectured by Armstrong [5, Conjecture 2.6] and which should be compared with (20).

Theorem 30. Suppose $s$ and $t$ are coprime. The average size of an $(s, t)$-core partition is

$$
\frac{(s-1)(t-1)(s+t+1)}{24} .
$$

The case $(s, t)=(s, s+1)$ had been established by Stanley and Zanello [17]. On the other hand, employing results by Ford, Mai and Sze [9] on self-conjugate core partitions, Chen, Huang and Wang [8] showed that the average size of a self-conjugate $(s, t)$-core partitions is also given by (21).

Xiong [19] proved the following result for $(s, s+1)$-core partitions into distinct parts, which had been conjectured by Amdeberhan [1].

Theorem 31. The total sum of the sizes of $(s, s+1)$-core partitions into distinct parts is

$$
\sum_{\substack{i+j+k=s+1 \\ i, j, k \geqslant 1}} F_{i} F_{j} F_{k} .
$$

Note that the total sum (22), divided by the number $F_{s+1}$ of such partitions, gives the average size of $(s, s+1)$-core partitions into distinct parts. Also note that, by general properties of constant recursive sequences, the convolution sum (22) of Fibonacci numbers can expressed in the simpler but possibly less illuminating form $\frac{1}{50}\left((5 s+7) s F_{s+1}-6(s+\right.$ 
1) $\left.F_{s}\right)$. This result was generalized by Zaleski [22] to higher moments of the sizes of $(s, s+$ 1)-core partitions into distinct parts. In subsequent work, Zaleski [23] also considered moments of the sizes of $(s, m s-1)$-core partition into distinct parts. For recent results on the largest sizes of $(s, m s \pm 1)$-core partitions into distinct parts, we refer to [20].

As in Theorem 2, let $f_{s}(q)=C_{s, m, r}^{d}(q)$ denote the generating polynomial for the number of parts of $(s, m s+r)$-core partitions into $d$-distinct parts. Clearly, $n_{s}=f_{s}(1)$ is the number of these partitions. On the other hand, note that $p_{s}=f_{s}^{\prime}(1)$ is the total number of parts of these partitions. We therefore obtain the following result as a consequence of Theorem 2.

Corollary 32. Suppose the conditions of Theorem 2 hold. Then $p_{s}$, the total sum of the numbers of parts of $(s, m s+r)$-core partitions into d-distinct parts, satisfies

$$
p_{s}=p_{s-1}+m p_{s-d-1}+\left(\begin{array}{c}
m+1 \\
2
\end{array}\right) n_{s-d-1} .
$$

Note that $p_{s} / n_{s}$ is the average number of parts of these partitions. Since the $n_{s}$ are constant recursive by Theorem 2 , it follows that the $p_{s}$ are constant recursive as well. We illustrate this in the particularly pleasing cases of $(s, s+1)$-core partitions into distinct parts (in which case $\left.n_{s}=F_{s+1}\right)$ as well as $(s, 2 s-1$ )-core partitions into distinct parts (in which case $n_{s}=2^{s-1}$, as noted in Example 21).

Corollary 33. The total sum of the numbers of parts of $(s, s+1)$-core partitions into distinct parts is

$$
\sum_{\substack{i+j=s \\ i, j \geqslant 1}} F_{i} F_{j}=\frac{1}{5}\left(2 s F_{s+1}-(s+1) F_{s}\right) .
$$

Observe the surprising similarity of (23) with (22), the total sum of the sizes of $(s, s+$ 1)-core partitions into distinct parts.

Corollary 34. The total sum of the numbers of parts of $(s, 2 s-1)$-core partitions into distinct parts is $(s-1) 2^{s-2}$. The number of these partitions is $2^{s-1}$.

Likewise, the higher moments for the number of parts of $(s, m s+r)$-core partitions into $d$-distinct parts can be obtained from higher derivatives of the generating polynomial $f_{s}(q)=C_{s, m, r}^{d}(q)$.

\section{Conclusion}

Under certain restrictions on $s$ and $t$, we have determined the generating polynomials $f_{s, t}(q)$ for $(s, t)$-core partitions into $d$-distinct parts, where $q$ keeps track of the number of parts. It would be desirable, but appears difficult, to prove enumeration results for general $s$ and $t$. It would also be desirable, but again appears difficult, to be able to incorporate the sizes of the partitions in the generating polynomials. More generally, it is 
natural to ask whether we can include additional statistics (besides the number of parts) to the enumeration results for core partitions (see, for instance, the final section of [15] for a discussion of including the bounce statistic on $(s, m s+1)$-core partitions).

The natural question whether Anderson's [2] result (1) that the number of $(s, t)$-core partitions is given by the generalized Catalan numbers can be similarly extended to keep track of additional statistics, like the number of parts, leads to intriguing and surprisingly difficult open problems. For instance, it is an open problem (proposed by Dennis Stanton; see [5]) to find a statistic stat $(\lambda)$ on $(s, t)$-core partitions $\lambda$ such that, for coprime $s$ and $t$

$$
\sum_{\lambda} q^{\operatorname{stat}(\lambda)}=\frac{1}{[s+t]_{q}}\left(\begin{array}{c}
s+t \\
s
\end{array}\right)_{q}
$$

where the sum is over all $(s, t)$-core partitions $\lambda$ and where the right-hand side are the usual $q$-analogs. Interestingly, Armstrong, Hanusa and Jones [5, Conjecture 2.8] provide a conjectural candidate for such a statistic, which is the sum of $n(\lambda)$, the number of parts of $\lambda$, plus a second (nonnegative) statistic $m(\lambda)$, the skew length of $\lambda$. Anderson established (1) by exhibiting a bijection between the set of $(s, t)$-core partitions and the set of $(s, t)$ Dyck paths (lattice paths from one corner of a $s \times t$ rectangle to the opposite corner, which stay above the diagonal connecting these corners). In particular, statistics on core partitions can be obtained from statistics on Dyck paths. As an indication that proving (24), a q-analog of Anderson's enumeration (1), is a difficult problem, we mention that even the nonnegativity of the coefficients of the generalized $q$-Catalan numbers on the right-hand side of (24) is nontrivial and that no elementary proof is known. On the other hand, in the words of [5], the conjecture that (24) holds for stat $(\lambda)=n(\lambda)+m(\lambda)$ is "just a shadow from the more general subject of $q, t$-Catalan combinatorics" (below, we will use the letter $x$ in place of $t$ ). A particularly appealing general conjecture is that

$$
\sum_{\lambda} q^{n(\lambda)} x^{m(\lambda)}=\sum_{\lambda} q^{m(\lambda)} x^{n(\lambda)}
$$

where both sums are over $(s, t)$-core partitions $\lambda$ for coprime $s$ and $t$. Here, the left-hand side defines the $q, x$-Catalan numbers. For more details and further references, we refer to [5], as well as [10] where special cases of the symmetry (25) are proved.

As noted in Example 21, it follows from $\boldsymbol{C}_{s, 2,-1}(q)=(1+q)^{s-1}$ that the number of $(s, 2 s-1)$-core partitions into $k$ distinct parts is $\left(\begin{array}{c}s-1 \\ k\end{array}\right)$. Less generally, as observed in [18], there are $2^{s-1}$ many $(s, 2 s-1)$-core partitions into distinct parts. On the other hand, Yan, Qin, Jin, Zhou [21], Zaleski, Zeilberger [24], Baek, Nam, Yu [6], and Paramonov [15] show that, for odd $s$, the number of $(s, s+2)$-core partitions into distinct parts is $2^{s-1}$ as well. In our notation (2),

$$
\boldsymbol{C}_{s, 1,2}(1)=2^{s-1} \text {. }
$$

It would be interesting to determine an explicit formula for $\boldsymbol{C}_{s, 1,2}(q)=\sum c_{n}(s) q^{n}$, a $q$-analog of (26), and thus obtain the number of such partitions into $k$ parts. Limited numerical data suggests that $c_{n}(s)$, for $s \geqslant n$, is a polynomial in $s$ of degree $n$. 


\section{Acknowledgements}

We thank Huan Xiong for sending a preprint of [20] and for mentioning Zaleski's conjectures [23] on the moments of the size of an $(n, d n-1)$-core partition into distinct parts. We are also grateful to the reviewers for their careful reading and helpful suggestions.

\section{References}

[1] T. Amdeberhan. Theorems, problems and conjectures. Preprint, 2015. arXiv:1207.4045, (updated version available at http://math.tulane.edu/ tamdeberhan/conjectures.html).

[2] J. Anderson. Partitions which are simultaneously $t_{1^{-}}$and $t_{2}$-core. Discrete Mathematics, 248(1-3):237-243, 2002.

[3] G. E. Andrews. The Theory of Partitions. Addison-Wesley, 1976.

[4] G. E. Andrews and K. Eriksson. Integer Partitions. Cambridge University Press, 2004.

[5] D. Armstrong, C. R. H. Hanusa, and B. C. Jones. Results and conjectures on simultaneous core partitions. European Journal of Combinatorics, 41:205-220, 2014.

[6] J. Baek, H. Nam, and M. Yu. A bijective proof of Amdeberhan's conjecture on the number of $(s, s+2)$-core partitions with distinct parts. Discrete Mathematics, 341(5):1294-1300, 2018.

[7] M. Beck and S. Robins. Computing the Continuous Discretely: Integer-Point Enumeration in Polyhedra. Springer, New York, 2007.

[8] W. Chen, H. Huang, and L. Wang. Average size of a self-conjugate $(s, t)$-core partition. Proceedings of the American Mathematical Society, 144(4):1391-1399, 2016.

[9] B. Ford, H. Mai, and L. Sze. Self-conjugate simultaneous $p$ - and $q$-core partitions and blocks of $A_{n}$. Journal of Number Theory, 129(4):858-865, 2009.

[10] E. Gorsky and M. Mazin. Compactified Jacobians and $q$,t-Catalan numbers, II. Journal of Algebraic Combinatorics, 39(1):153-186, 2014.

[11] P. Johnson. Lattice points and simultaneous core partitions. The Electronic Journal of Combinatorics, 25(3):\#P3.47, 2018.

[12] N. Kravitz. On the number of simultaneous core partitions with $d$-distinct parts. Discrete Mathematics, 342(12):\#111592, 14 p., 2019.

[13] R. Nath and J. A. Sellers. Abaci structures of $(s, m s \pm 1)$-core partitions. The Electronic Journal of Combinatorics, 24(1):\#P1.5, 2017.

[14] J. B. Olsson and D. Stanton. Block inclusions and cores of partitions. Aequationes Mathematicae, 74(1-2):90-110, 2007.

[15] K. Paramonov. Cores with distinct parts and bigraded Fibonacci numbers. Discrete Mathematics, 341(4):875-888, 2018. 
[16] M. Sahin. Core partitions with $d$-distinct parts. Australasian Journal of Combinatorics, 72(1):19-28, 2018.

[17] R. P. Stanley and F. Zanello. The Catalan case of Armstrong's conjecture on simultaneous core partitions. SIAM Journal on Discrete Mathematics, 29(1):658-666, 2015 .

[18] A. Straub. Core partitions into distinct parts and an analog of Euler's theorem. European Journal of Combinatorics, 57:40-49, 2016.

[19] H. Xiong. Core partitions with distinct parts. The Electronic Journal of Combinatorics, 25(1):\#P1.57, 2018.

[20] H. Xiong. On the largest sizes of certain simultaneous core partitions with distinct parts. European Journal of Combinatorics, 71:33-42, 2018.

[21] S. H. F. Yan, G. Qin, Z. Jin, and R. D. P. Zhou. On $(2 k+1,2 k+3)$-core partitions with distinct parts. Discrete Mathematics, 340(6):1191-1202, 2017.

[22] A. Zaleski. Explicit expressions for the moments of the size of an $(s, s+1)$-core partition with distinct parts. Advances in Applied Mathematics, 84:1-7, 2017.

[23] A. Zaleski. Explicit expressions for the moments of the size of an $(n, d n-1)$-core partition with distinct parts. Integers, 19:\#A26, 11 p., 2019.

[24] A. Zaleski and D. Zeilberger. Explicit expressions for the expectation, variance and higher moments of the size of a $(2 n+1,2 n+3)$-core partition with distinct parts. Journal of Difference Equations and Applications, 23(7):1241-1254, 2017. 